PROCEEDINGS OF THE

AMERICAN MATHEMATICAL SOCIETY

Volume 136, Number 8, August 2008, Pages 2911-2920

S 0002-9939(08)09416-1

Article electronically published on April 11, 2008

\title{
ESTIMATES OF GROMOV'S BOX DISTANCE
}

\author{
KEI FUNANO \\ (Communicated by Jon G. Wolfson) \\ This paper is dedicated to our advisors.
}

\begin{abstract}
In 1999, M. Gromov introduced the box distance function $\square_{\lambda}$ on the space of all mm-spaces. In this paper, by using the method of $\mathrm{T}$. H. Colding, we estimate $\square_{\lambda}\left(\mathbb{S}^{n}, \mathbb{S}^{m}\right)$ and $\square_{\lambda}\left(\mathbb{C} P^{n}, \mathbb{C} P^{m}\right)$, where $\mathbb{S}^{n}$ is the $n$-dimensional unit sphere in $\mathbb{R}^{n+1}$ and $\overline{\mathbb{C}} P^{n}$ is the $n$-dimensional complex projective space equipped with the Fubini-Study metric. In particular, we give the complete answer to an exercise of Gromov's green book. We also estimate $\square_{\lambda}(S O(n), S O(m))$ from below, where $S O(n)$ is the special orthogonal group.
\end{abstract}

\section{INTRODUCTION}

In 1999, M. Gromov developed the theory of mm-spaces in [4, Chapter $3 \frac{1}{2}{ }_{+}$] by introducing two distance functions, called the box distance function $\square_{\lambda}$ and the observable distance function $\underline{H}_{\lambda} \mathcal{L} \iota_{1}$, on the space $\mathcal{X}$ of all isomorphic classes of mm-spaces. Here, an mm-space is a triple $\left(X, d_{X}, \mu_{X}\right)$, where $d_{X}$ is a complete separable metric on a set $X$ and $\mu_{X}$ a finite Borel measure on $\left(X, d_{X}\right)$. The notion of the distance function $\square_{\lambda}$ is considered as a natural extension of the Gromov-Hausdorff distance function to the space $\mathcal{X}$. On the other hand, the notion of the distance function $\underline{H}_{\lambda} \mathcal{L} \iota_{1}$ is related to measure concentration. Roughly speaking, "measure concentration" amounts to saying that the push-forward measures $f_{n *}\left(\mu_{n}\right)$ on $\mathbb{R}$ concentrate to a point for any sequence of 1-Lipschitz functions $f_{n}:\left(X_{n}, d_{n}, \mu_{n}\right) \rightarrow \mathbb{R}$. For instance, the unit spheres in Euclidean spaces $\left\{\mathbb{S}^{n}\right\}_{n=1}^{\infty}$, the complex projective spaces $\left\{\mathbb{C} P^{n}\right\}_{n=1}^{\infty}$ equipped with the Fubini-Study metrics, and the special orthogonal groups $\{S O(n)\}_{n=1}^{\infty}$ have this property (we suppose that each space is equipped with its Riemannian volume measure normalized to have total volume 1). Gromov defined the distance $\underline{H}_{\lambda} \mathcal{L} \iota_{1}(X, Y)$ by using the Hausdorff distance between the space of 1-Lipschitz functions on $X$ and that on $Y$, and showed that a sequence $\left\{X_{n}\right\}_{n=1}^{\infty}$ of mm-spaces concentrates if and only if the sequence $\left\{X_{n}\right\}_{n=1}^{\infty}$ converges to a one-point space with respect to the distance function $\underline{H}_{\lambda} \mathcal{L} \iota_{1}$.

Received by the editors June 18, 2007.

2000 Mathematics Subject Classification. Primary 28E99, 53C23.

Key words and phrases. mm-space, box distance function, observable distance function.

This work was partially supported by research fellowships of the Japan Society for the Promotion of Science for Young Scientists.

(C)2008 American Mathematical Society Reverts to public domain 28 years from publication 
The topology on $\mathcal{X}$ determined by $\square_{\lambda}$ is strictly stronger than that of $\underline{H}_{\lambda} \mathcal{L} \iota_{1}$. In fact, the sequences $\left\{\mathbb{S}^{n}\right\}_{n=1}^{\infty},\left\{\mathbb{C} P^{n}\right\}_{n=1}^{\infty}$, and $\{S O(n)\}_{n=1}^{\infty}$ are all divergent with respect to the distance $\square_{\lambda}$ (see Proposition 3.1). This is related to the following exercise in Gromov's book:

Exercise (cf. [4, Section $\left.3 \frac{1}{2} \cdot 18\right]$ ). Estimate the distance $\square_{\lambda}\left(\mathbb{S}^{n}, \mathbb{S}^{m}\right)$.

To solve the exercise, applying a method of [1, Lemma 5.10], we will estimate $\square_{\lambda}(M, N)$ from below for compact Riemannian manifolds $M$ and $N$ with positive Ricci curvatures and the volume measures satisfying a homogeneity condition (see Lemma 3.4). As a result, we get the following proposition:

Proposition 1.1. Assume that two sequences $\left\{n_{k}\right\}_{k=1}^{\infty},\left\{m_{k}\right\}_{k=1}^{\infty}$ of natural numbers satisfy $n_{k} \leq C_{1} k, m_{k} \leq C_{2} k$ and $\left|n_{k}-m_{k}\right| \geq C_{3} k, k=1,2, \cdots$, for some positive constants $C_{1}, C_{2}, C_{3}$. Then, we have

$$
\liminf _{k \rightarrow \infty} \square_{1}\left(\mathbb{S}^{n_{k}}, \mathbb{S}^{m_{k}}\right), \liminf _{k \rightarrow \infty} \square_{1}\left(\mathbb{C} P^{n_{k}}, \mathbb{C} P^{m_{k}}\right) \geq \min \left\{2^{-\frac{C_{1}}{C_{3}}} \pi^{-\frac{C_{2}}{C_{3}}}, 2^{-\frac{C_{2}}{C_{3}}} \pi^{-\frac{C_{1}}{C_{3}}}\right\} .
$$

In particular, if in addition $\left|n_{k}-m_{k}\right| \geq C_{4} k^{\alpha}, k=1,2, \cdots$, holds for some constant $C_{4}>0$ and a number $\alpha>1$, then we have

$$
\lim _{k \rightarrow \infty} \square_{1}\left(\mathbb{S}^{n_{k}}, \mathbb{S}^{m_{k}}\right), \lim _{k \rightarrow \infty} \square_{1}\left(\mathbb{C} P^{n_{k}}, \mathbb{C} P^{m_{k}}\right)=1 .
$$

Note that $\operatorname{diam}\left(\mathcal{X}_{1}, \square_{1}\right)=1$, where $\mathcal{X}_{1}$ is the space of all mm-spaces with Borel probability measures.

We estimate $\square_{\lambda}(S O(n), S O(m))$ from below by the difference of their diameters (see Lemma 3.8). Consequently, we obtain the following proposition:

Proposition 1.2. Assume that two sequences $\left\{n_{k}\right\}_{k=1}^{\infty},\left\{m_{k}\right\}_{k=1}^{\infty}$ of natural numbers satisfy $n_{k} \leq C_{1} k, m_{k} \leq C_{2} k$ and $\left|n_{k}-m_{k}\right| \geq C_{3} \sqrt{k}, k=1,2, \cdots$, for some positive constants $C_{1}, C_{2}, C_{3}$. Then, we have

$$
\liminf _{k \rightarrow \infty} \unrhd_{1}\left(S O\left(n_{k}\right), S O\left(m_{k}\right)\right) \geq \min \left\{\frac{1}{2}, \frac{C_{3}}{\sqrt{C_{1}}+\sqrt{C_{2}}}\right\} .
$$

In particular, if in addition $\left|n_{k}-m_{k}\right| \geq C_{4} k^{\alpha}, k=1,2, \cdots$, holds for some constant $C_{4}>0$ and a number $\alpha>1 / 2$, then we have

$$
\liminf _{k \rightarrow \infty} \square_{1}\left(S O\left(n_{k}\right), S O\left(m_{k}\right)\right) \geq \frac{1}{2} .
$$

As it is related to the above Gromov's exercise, we also prove the following proposition. This proposition is also mentioned by Gromov in [4, Section $3 \frac{1}{2} \cdot 3$, Exercise $(e)]$.

Proposition 1.3. We have

$$
\square_{1}\left(\mathbb{S}^{n}, \mathbb{S}^{n-1}\right), \square_{1}\left(\mathbb{C} P^{n}, \mathbb{C} P^{n-1}\right) \rightarrow 0
$$

as $n \rightarrow \infty$.

\section{Preliminaries}

\subsection{Definition of Gromov's box distance function $\square_{\lambda}$.}

Definition 2.1. Let $\lambda \geq 0$ and $(X, \mu)$ be a measure space with $\mu(X)<+\infty$. For two maps $d_{1}, d_{2}: X \times X \rightarrow \mathbb{R}$, we define a number $\square_{\lambda}\left(d_{1}, d_{2}\right)$ as the infimum of $\varepsilon>0$ such that there exists a measurable subset $T_{\varepsilon} \subseteq X$ of measure at least $\mu(X)-\lambda \varepsilon$ satisfying $\left|d_{1}(x, y)-d_{2}(x, y)\right| \leq \varepsilon$ for any $x, y \in T_{\varepsilon}$. 
It is easy to see that this is a distance function on the set of all functions on $X \times X$, and the two distance functions $\square_{\lambda}$ and $\square_{\lambda^{\prime}}$ are equivalent to each other for any $\lambda, \lambda^{\prime}>0$.

Definition 2.2 (parameter). Let $X$ be an mm-space and $\mu(X)=m$. Then, there exists a Borel measurable map $\varphi:[0, m] \rightarrow X$ with $\varphi_{*}(\mathcal{L})=\mu$, where $\mathcal{L}$ stands for the Lebesgue measure on $[0, m]$. We call $\varphi$ a parameter of $X$.

Note that if the support of $X$ is not one-point, then its parameter is not unique.

Definition 2.3 (Gromov's box distance function). If two mm-spaces $X, Y$ satisfy $\mu_{X}(X)=\mu_{Y}(Y)=m$, we define

$$
\square_{\lambda}(X, Y):=\inf \square_{\lambda}\left(\varphi_{X}^{*} d X, \varphi_{Y}^{*} d_{Y}\right),
$$

where the infimum is taken over all parameters $\varphi_{X}:[0, m] \rightarrow X, \varphi_{Y}:[0, m] \rightarrow Y$, and $\varphi_{X}^{*} d X$ is defined by $\varphi_{X}^{*} d_{X}(s, t):=d_{X}\left(\varphi_{X}(s), \varphi_{X}(t)\right)$ for $s, t \in[0, m]$. If $\mu_{X}(X)<\mu_{Y}(Y)$, putting $m:=\mu_{X}(X), m^{\prime}:=\mu_{Y}(Y)$, we define

$$
\square_{\lambda}(X, Y):=\square_{\lambda}\left(X, \frac{m}{m^{\prime}} Y\right)+m^{\prime}-m,
$$

where $\left(m / m^{\prime}\right) Y:=\left(Y, d_{Y},\left(m / m^{\prime}\right) \mu_{Y}\right)$.

We recall that two mm-spaces are isomorphic to each other if there is a measure preserving isometry between the supports of their measures. $\square_{\lambda}$ is a distance function on $\mathcal{X}$ for any $\lambda \geq 0$. See [2, Sections 1,3] for a complete proof of that. Note that the distance functions $\square_{\lambda}$ and $\square_{\lambda^{\prime}}$ are equivalent to each other for distinct $\lambda, \lambda^{\prime}>0$.

2.2. Definition of observable distance functions $\underline{H}_{\lambda} \mathcal{L} \iota_{1}$. For a measure space $(X, \mu)$ with $\mu(X)<+\infty$, we denote by $\mathcal{F}(X, \mathbb{R})$ the space of all functions on $X$. Given $\lambda \geq 0$ and $f, g \in \mathcal{F}(X, \mathbb{R})$, we put

$$
\operatorname{me}_{\lambda}(f, g):=\inf \{\varepsilon>0 \mid \mu(\{x \in X|| f(x)-g(x) \mid \geq \varepsilon\}) \leq \lambda \varepsilon\} .
$$

Note that this me $_{\lambda}$ is a distance function on $\mathcal{F}(X, \mathbb{R})$ for any $\lambda \geq 0$, and its topology on $\mathcal{F}(X, \mathbb{R})$ coincides with the topology of the convergence in measure for any $\lambda>0$. Also, the distance functions me $_{\lambda}$ for all $\lambda>0$ are mutually equivalent.

We recall that the Hausdorff distance between two closed subsets $A$ and $B$ in a metric space $X$ is defined by

$$
d_{H}(A, B):=\inf \left\{\varepsilon>0 \mid A \subseteq B_{\varepsilon}, B \subseteq A_{\varepsilon}\right\},
$$

where $A_{\varepsilon}$ is a closed $\varepsilon$-neighborhood of $A$.

Let $(X, \mu)$ be a measure space with $\mu(X)<+\infty$. For a semi-distance $d$ on $X$, we indicate by $\mathcal{L i p}_{1}(d)$ the space of all 1-Lipschitz functions on $X$ with respect to $d$. Note that $\mathcal{L}_{i p_{1}}(d)$ is a closed subset in $\left(\mathcal{F}(X, \mathbb{R})\right.$, me $\left._{\lambda}\right)$ for any $\lambda \geq 0$.

Definition 2.4. For $\lambda \geq 0$ and two semi-distance functions $d, d^{\prime}$ on $X$, we define

$$
H_{\lambda} \mathcal{L}_{\iota_{1}}\left(d, d^{\prime}\right):=d_{H}\left(\mathcal{L} i p_{1}(d), \mathcal{L} i p_{1}\left(d^{\prime}\right)\right),
$$

where $d_{H}$ stands for the Hausdorff distance function in $\left(\mathcal{F}(X, \mathbb{R}), \mathrm{me}_{\lambda}\right)$.

This $H_{\lambda} \mathcal{L}_{\iota_{1}}$ is actually a distance function on the space of all semi-distance functions on $X$ for all $\lambda \geq 0$, and the two distance functions $H_{\lambda} \mathcal{L} \iota_{1}$ and $H_{\lambda^{\prime}} \mathcal{L} \iota_{1}$ are equivalent to each other for any $\lambda, \lambda^{\prime}>0$. 
Lemma 2.5. For any two semi-distance functions $d, d^{\prime}$ on $X$, we have

$$
H_{\lambda} \mathcal{L}_{1}\left(d, d^{\prime}\right) \leq \square_{\lambda}\left(d, d^{\prime}\right) .
$$

Proof. For any $\varepsilon>0$ with $\square_{\lambda}(X, Y)<\varepsilon$, there exists a measurable subset $T_{\varepsilon} \subseteq X$ such that $\mu\left(X \backslash T_{\varepsilon}\right) \leq \lambda \varepsilon$ and $\left|d(x, y)-d^{\prime}(x, y)\right| \leq \varepsilon$ for any $x, y \in T_{\varepsilon}$. Given arbitrary $f \in \mathcal{L} \operatorname{Lip}_{1}(d)$, we define $\widetilde{f} \in \mathcal{F}(X, \mathbb{R})$ by $\widetilde{f}(x):=\inf \left\{f(y)+d^{\prime}(x, y) \mid y \in\right.$ $\left.T_{\varepsilon}\right\}$. We easily see that $\tilde{f} \in \mathcal{L} i p_{1}\left(d^{\prime}\right)$ and $\widetilde{f}(x) \leq f(x)$ for any $x \in T_{\varepsilon}$. Taking any $x \in T_{\varepsilon}$, we have

$$
\begin{aligned}
|f(x)-\tilde{f}(x)| & =f(x)-\tilde{f}(x) \\
& =\sup \left\{f(x)-f(y)-d^{\prime}(x, y) \mid y \in T_{\varepsilon}\right\} \\
& \leq \sup \left\{d(x, y)-d^{\prime}(x, y) \mid y \in T_{\varepsilon}\right\} \\
& \leq \varepsilon .
\end{aligned}
$$

Therefore, we get $\operatorname{me}_{\lambda}(f, \widetilde{f}) \leq \varepsilon$, which implies $\mathcal{L}_{i} p_{1}(d) \subseteq\left(\mathcal{L}_{i} p_{1}\left(d^{\prime}\right)\right)_{\varepsilon}$. Similarly,

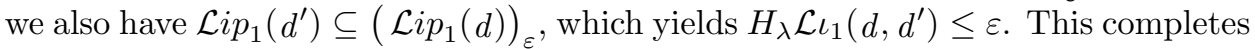
the proof.

Definition 2.6 (Observable distance function). If two mm-spaces $X, Y$ satisfy $\mu_{X}(X)=\mu_{Y}(Y)=m$, we define

$$
\underline{H}_{\lambda} \mathcal{L} \iota_{1}(X, Y):=\inf H_{\lambda} \mathcal{L} \iota_{1}\left(\varphi_{X}^{*} d X, \varphi_{Y}^{*} d_{Y}\right),
$$

where the infimum is taken over all parameters $\varphi_{X}:[0, m] \rightarrow X, \varphi_{Y}:[0 . m] \rightarrow Y$. If $\mu_{X}(X)<\mu_{Y}(Y)$, putting $m:=\mu_{X}(X), m^{\prime}:=\mu_{Y}(Y)$, we define

$$
\underline{H}_{\lambda} \mathcal{L} \iota_{1}(X, Y):=\underline{H}_{\lambda} \mathcal{L} \iota_{1}\left(X, \frac{m}{m^{\prime}} Y\right)+m^{\prime}-m .
$$

Hence, $\underline{H}_{\lambda} \mathcal{L} \iota_{1}$ is a distance function on $\mathcal{X}$ for any $\lambda \geq 0$. See [2, Section 3] for a complete proof. Note that the distance functions $\underline{H}_{\lambda} \mathcal{L} \iota_{1}$ and $\underline{H}_{\lambda^{\prime}} \mathcal{L} \iota_{1}$ are equivalent to each other for any $\lambda, \lambda^{\prime}>0$.

For a Borel measure $\nu$ on $\mathbb{R}$ with $m:=\nu(\mathbb{R})<+\infty$ and $\kappa>0$, we put $\operatorname{diam}(\nu, m-\kappa):=\inf \left\{\operatorname{diam} Y \mid Y \subseteq \mathbb{R}\right.$ is a Borel subset such that $\left.\nu_{Y}(Y) \geq m-\kappa\right\}$, and call it the partial diameter on $\nu$.

Definition 2.7 (Observable diameter). Let $(X, d, \mu)$ be an mm-space and let $m:=$ $\mu(X)$. For any $\kappa>0$ we define the observable diameter of $X$ by

$$
\begin{aligned}
\operatorname{diam}\left(X \stackrel{\operatorname{Lip}_{1}}{\longrightarrow}\right. & \mathbb{R}, m-\kappa) \\
& :=\sup \left\{\operatorname{diam}\left(f_{*}(\mu), m-\kappa\right) \mid f: X \rightarrow \mathbb{R} \text { is a 1-Lipschitz function }\right\} .
\end{aligned}
$$

The idea of the observable diameter comes from quantum and statistical mechanics; that is, we think of $\mu$ as a state on a configuration space $X$ and $f$ is interpreted as an observable. We define a sequence $\left\{X_{n}\right\}_{n=1}^{\infty}$ of mm-spaces as a Lévy family if $\operatorname{diam}\left(X_{n} \stackrel{\text { Lip }_{1}}{\longrightarrow} \mathbb{R}, m_{n}-\kappa\right) \rightarrow 0$ as $n \rightarrow \infty$ for any $\kappa>0$, where $m_{n}$ is the total measure of the mm-space $X_{n}$. This is equivalent to the fact that for any $\varepsilon>0$ and any sequence $\left\{f_{n}: X_{n} \rightarrow \mathbb{R}\right\}_{n=1}^{\infty}$ of 1-Lipschitz functions, we have

$$
\mu_{n}\left(\left\{x \in X_{n}|| f_{n}(x)-m_{f_{n}} \mid \geq \varepsilon\right\}\right) \rightarrow 0 \text { as } n \rightarrow \infty,
$$

where $m_{f_{n}}$ is some constant determined by $f_{n}$. 
For a compact connected Riemannian manifold $M$, we denote by $\mu_{M}$ the Riemannian volume measure of $M$ normalized as $\mu_{M}(M)=1$ and by $d_{M}$ the Riemannian distance of $M$. We shall consider $M$ as an mm-space $\left(M, d_{M}, \mu_{M}\right)$.

Example 2.8. Let $\left\{M_{n}\right\}_{n=1}^{\infty}$ be a sequence of compact connected Riemannian manifolds and assume that $\operatorname{Ric}_{M_{n}} \geq \kappa_{n} \rightarrow+\infty$ as $n \rightarrow \infty$. Then, by virtue of Lévy-Gromov's isoperimetric inequality, the sequence $\left\{M_{n}\right\}_{n=1}^{\infty}$ is a Lévy family (cf. [5, Section 1, Remark 2]). For example, $\left\{\mathbb{S}^{n}\right\}_{n=1}^{\infty}$ and $\left\{\mathbb{C} P^{n}\right\}_{n=1}^{\infty}$ are Lévy families. Recall that the Fubini-Study metric on $\mathbb{C} P^{n}$ is the unique Riemannian metric on $\mathbb{C} P^{n}$ such that the canonical projection $\mathbb{S}^{2 n+1} \rightarrow \mathbb{C} P^{n}$ is a Riemannian submersion. Since $\operatorname{Ric}_{S O(n)} \geq(n-1) / 4$, the sequence $\{S O(n)\}_{n=1}^{\infty}$ is a Lévy family. Since the distance function induced from the Hilbert-Schmidt norm on $S O(n)$ is not greater than that of the Riemannian distance function, $\{S O(n)\}_{n=1}^{\infty}$ is also a Lévy family with respect to the Hilbert-Schmidt norms.

Example 2.9 (Hamming cube). Let $\mu_{n}$ be the normalized counting measure on $\{0,1\}^{n}$ and $d_{n}$ be the Hamming distance function on $\{0,1\}^{n}$; that is,

$$
d_{n}\left(\left(x_{i}\right)_{i=1}^{n},\left(y_{i}\right)_{i=1}^{n}\right):=\frac{1}{n} \operatorname{Card}\left(\left\{i \in\{1, \cdots, n\} \mid x_{i} \neq y_{i}\right\}\right) .
$$

The mm-space $\{0,1\}^{n}$ is called the Hamming cube. The sequence $\left\{\{0,1\}^{n}\right\}_{n=1}^{\infty}$ is a Lévy family (cf. [4, Section $\left.3 \frac{1}{2} \cdot 42\right]$ ).

Gromov showed the following proposition by considering a constant $m_{f_{n}}$ in $(\diamond)$ as a Lipschitz function from a one-point space $\left\{*_{n}\right\}$ with total measure $\mu_{n}\left(X_{n}\right)$.

Proposition 2.10 (cf. [4, Section $\left.3 \frac{1}{2} .45\right]$ ). A sequence $\left\{X_{n}\right\}_{n=1}^{\infty}$ of mm-spaces is a Lévy family if and only if $\underline{H}_{\lambda} \mathcal{L}_{\iota_{1}}\left(X_{n},\left\{*_{n}\right\}\right) \rightarrow 0$ as $n \rightarrow \infty$ for any $\lambda>0$.

\section{Estimates of Gromov's box distance function}

Let $X$ be a metric space. Denote by $B_{X}(x, r)$ the closed ball in $X$ centered at $x \in X$ with radius $r>0$. A Borel measure $\mu$ on $X$ is said to be uniformly distributed if

$$
0<\mu\left(B_{X}(x, r)\right)=\mu\left(B_{X}(y, r)\right)<+\infty
$$

for any $r>0$ and $x, y \in X$.

From Lemma 2.5 we see that the topology on $\mathcal{X}$ determined by $\square_{\lambda}$ is not weaker than that of $\underline{H}_{\lambda} \mathcal{L} \iota_{1}$ for any $\lambda \geq 0$. For a Borel measure $\mu$ on a metric space, we denote by $\operatorname{Supp} \mu$ its support.

Proposition 3.1. Let $\left\{\left(X_{n}, d_{n}, \mu_{n}\right)\right\}_{n=1}^{\infty}$ be a Lévy family such that $\mu_{n}$ is a uniformly distributed probability measure satisfying $X_{n}=\operatorname{Supp} \mu_{n}$ and $\inf _{n \in \mathbb{N}} \operatorname{diam} X_{n}>$ 0 . Then, the sequence $\left\{X_{n}\right\}_{n=1}^{\infty}$ does not converge with respect to the distance function $\square_{\lambda}$ for any $\lambda \geq 0$.

Proof. Suppose that $\left\{X_{n}\right\}_{n=1}^{\infty}$ converges and let $X$ be its limit. Since $\left\{X_{n}\right\}_{n=1}^{\infty}$ is a Lévy family, by using Proposition 2.10, $X$ must be a one-point space. Fix $\varepsilon>0$ with $\varepsilon<\min \left\{3, \inf _{n \in \mathbb{N}} \operatorname{diam} X_{n}\right\} / 3$. For any sufficiently large $n \in \mathbb{N}$, there exist a parameter $\varphi_{n}:[0,1] \rightarrow X_{n}$ of $X_{n}$ and a Borel subset $T_{n} \subseteq[0,1]$ such that $\mathcal{L}\left(T_{n}\right)>1-\varepsilon / 2$ and $d_{n}\left(\varphi_{n}(s), \varphi_{n}(t)\right)<\varepsilon / 2$ for any $s, t \in T_{n}$. Fix a point $t_{n} \in T_{n}$. 
There exists a point $x_{n} \in X_{n}$ such that $d_{n}\left(\varphi_{n}\left(t_{n}\right), x_{n}\right) \geq \operatorname{diam} X_{n} / 3>\varepsilon$, and hence $B_{X_{n}}\left(\varphi_{n}\left(t_{n}\right), \varepsilon / 2\right) \cap B_{X_{n}}\left(x_{n}, \varepsilon / 2\right)=\emptyset$. Therefore, we get

$$
\begin{aligned}
1 & \geq \mu_{n}\left(B_{X_{n}}\left(\varphi_{n}\left(t_{n}\right), \varepsilon / 2\right) \cup B_{X_{n}}\left(x_{n}, \varepsilon / 2\right)\right) \\
& =2 \mu_{n}\left(B_{X_{n}}\left(\varphi_{n}\left(t_{n}\right), \varepsilon / 2\right)\right) \\
& =2 \mathcal{L}\left(\varphi_{n}^{-1}\left(B_{X_{n}}\left(\varphi_{n}\left(t_{n}\right), \varepsilon / 2\right)\right)\right) \geq 2 \mathcal{L}\left(T_{n}\right) \geq 2-\varepsilon>1,
\end{aligned}
$$

which gives a contradiction. This completes the proof.

From Proposition 3.1. we see that many Lévy families such as $\left\{\mathbb{S}^{n}\right\}_{n=1}^{\infty}$, $\left\{\mathbb{C} P^{n}\right\}_{n=1}^{\infty},\{S O(n)\}_{n=1}^{\infty}$, and $\left\{\{0,1\}^{n}\right\}_{n=1}^{\infty}$ have no convergent subsequences with respect to the distance function $\square_{\lambda}$. Therefore, the distance function $\square_{\lambda}$ determines the topology on $\mathcal{X}$ strictly stronger than that of the distance function $\underline{H}_{\lambda} \mathcal{L} \iota_{1}$ for any $\lambda>0$. However, since the proof of Proposition 3.1 is by contradiction, we do not estimate $\square_{\lambda}\left(X_{n}, X_{m}\right)$ from below for $n, m \in \mathbb{N}$.

The proof of the following lemma is an analogue of the proof of [1, Lemma 5.10].

Lemma 3.2. Let $\left(X, d_{X}, \mu_{X}\right),\left(Y, d_{Y}, \mu_{Y}\right)$ be mm-spaces and assume that $\mu_{X}, \mu_{Y}$ are uniformly distributed Borel probability measures. Denote by $v_{X}(r)$ (respectively, $v_{Y}(r)$ ) the measure of a closed ball of $X$ (respectively, $Y$ ) with radius $r>0$ and assume that $v_{X}(a+c) \leq(1-c) v_{Y}(a / 2)$ for some $a, c>0$ with $c<1$. Then, we have $\square_{1}(X, Y) \geq c$.

Proof. Let us prove the lemma by contradiction. Suppose that $\square_{1}(X, Y)<c$. Then, there exist a compact subset $T \subseteq[0,1]$ and two parameters $\varphi_{X}:[0,1] \rightarrow X$, $\varphi_{Y}:[0,1] \rightarrow Y$ such that

(1) $\mathcal{L}(T)>1-c$

(2) $\left.\varphi_{X}\right|_{T}: T \rightarrow X,\left.\varphi_{Y}\right|_{T}: T \rightarrow Y$ are continuous,

(3) $\left|d_{X}\left(\varphi_{X}(s), \varphi_{X}(t)\right)-d_{Y}\left(\varphi_{Y}(s), \varphi_{Y}(t)\right)\right|<c$ for any $s, t \in T$.

By (1) and (2), $\varphi_{Y}(T)$ is compact. Put

$$
\begin{aligned}
& l:=\max \left\{k \in \mathbb{N} \mid \text { there exist points } p_{i} \in \varphi_{Y}(T), i=1, \cdots, k,\right. \text { such that } \\
& \left.\qquad B_{Y}\left(p_{i}, a / 2\right) \cap B_{Y}\left(p_{j}, a / 2\right)=\emptyset \text { for any } i, j \text { with } i \neq j\right\} .
\end{aligned}
$$

Then, there exist points $p_{i} \in \varphi_{Y}(T), i=1, \cdots, l$, such that $B_{Y}\left(p_{i}, a / 2\right) \cap$ $B_{Y}\left(p_{j}, a / 2\right)=\emptyset$ for any $i, j$ with $i \neq j$. Hence, we get

$$
1 \geq \mu_{Y}\left(\bigcup_{i=1}^{l} B_{Y}\left(p_{i}, a / 2\right)\right)=\sum_{i=1}^{l} \mu_{Y}\left(B_{Y}\left(p_{i}, a / 2\right)\right)=l \cdot v_{Y}(a / 2) .
$$

It also follows from the definition of $l$ that $\varphi_{Y}(T) \subseteq \bigcup_{i=1}^{l} B_{Y}\left(p_{i}, a\right)$. For any $i=$ $1, \cdots, l$, we fix $t_{i} \in T$ with $p_{i}=\varphi_{Y}\left(t_{i}\right)$.

Claim 3.3.

$$
\varphi_{X}(T) \subseteq \bigcup_{i=1}^{l} B_{X}\left(\varphi_{X}\left(t_{i}\right), a+c\right)
$$


Proof. Take an arbitrary $q=\varphi_{X}(s) \in \varphi_{X}(T), s \in T$. Since $\varphi_{Y}(s) \in \varphi_{Y}(T) \subseteq$ $\bigcup_{i=1}^{l} B_{Y}\left(p_{i}, a\right)$, there exists $i$ with $1 \leq i \leq l$ such that $d_{Y}\left(\varphi_{Y}(s), p_{i}\right) \leq a$. Therefore, by using (2), we obtain

$$
d_{X}\left(\varphi_{X}(s), \varphi_{X}\left(t_{i}\right)\right)<d_{Y}\left(\varphi_{Y}(s), p_{i}\right)+c \leq a+c .
$$

This completes the proof of the claim.

Applying Claim 3.3 , we get

$$
1 \leq \sum_{i=1}^{l} \frac{\mu_{X}\left(B_{X}\left(\varphi_{X}\left(t_{i}\right), a+c\right)\right)}{\mu_{X}\left(\varphi_{X}(T)\right)}=l \cdot \frac{v_{X}(a+c)}{\mu_{X}\left(\varphi_{X}(T)\right)} \leq \frac{v_{X}(a+c)}{v_{Y}(a / 2) \cdot \mu_{X}\left(\varphi_{X}(T)\right)} .
$$

Since $\mu_{X}\left(\varphi_{X}(T)\right) \geq \mathcal{L}\left(\varphi_{X}^{-1}\left(\varphi_{X}(T)\right)\right) \geq \mathcal{L}(T)>1-c$, we obtain

$$
1 \leq \frac{v_{X}(a+c)}{v_{Y}(a / 2) \cdot \mu_{X}\left(\varphi_{X}(T)\right)}<\frac{v_{X}(a+c)}{v_{Y}(a / 2) \cdot(1-c)} \leq 1,
$$

which is a contradiction. This completes the proof of Lemma 3.2 .

For a compact Riemannian manifold $M$, we denote by $\operatorname{vol}(M)$ the total Riemannian volume of $M$. We indicate by $\Gamma$ the Gamma function.

Lemma 3.4. Let $M$ (respectively, $N$ ) be an $m$ (respectively, $n$ )-dimensional compact Riemannian manifold having a uniformly distributed Riemannian volume measure. Assume that $\operatorname{Ric}_{M} \geq(m-1) \kappa_{1}>0$ and Ric ${ }_{N}>0$, and put $a_{N}:=$ $\operatorname{vol}(N) / \operatorname{vol}\left(\mathbb{S}^{n}\right)$. If a positive number $c$ with $c<1$ satisfies

$$
c^{n-m} \leq(1-c) \frac{n a_{N}\left(\kappa_{1}\right)^{m / 2} \Gamma\left(\frac{m+1}{2}\right) \Gamma\left(\frac{n}{2}\right)}{m 2^{n+1} \pi^{m-1} \Gamma\left(\frac{m}{2}\right) \Gamma\left(\frac{n+1}{2}\right)} \text { and } c \sqrt{\kappa_{1}} \leq \pi,
$$

then we have $\square_{1}(M, N) \geq c$.

Proof. For $r>0$, we put $v_{M}(r):=\mu_{M}\left(B_{M}(x, r)\right)$ for $x \in M$ and $v_{N}(r):=$ $\mu_{N}\left(B_{N}(y, r)\right)$ for $y \in N$. From the Bishop-Gromov volume comparison theorem, we get

$v_{M}(c / 2)=\frac{\operatorname{vol}\left(B_{M}(x, c / 2)\right)}{\operatorname{vol}(M)} \geq v_{\mathbb{S}^{m}}\left(\left(c \sqrt{\kappa_{1}}\right) / 2\right)=\frac{\operatorname{vol}\left(\mathbb{S}^{m-1}\right)}{\operatorname{vol}\left(\mathbb{S}^{m}\right)} \int_{0}^{\left(c \sqrt{\kappa_{1}}\right) / 2} \sin ^{m-1} \theta d \theta$.

From $c \sqrt{\kappa_{1}} \leq \pi$, we have $\sin \theta \geq(\pi \theta) / 2$ for any $\theta \in\left[0,\left(c \sqrt{\kappa_{1}}\right) / 2\right]$. Hence, we obtain

$$
v_{M}(c / 2) \geq \frac{2^{m-1} \operatorname{vol}\left(\mathbb{S}^{m-1}\right)}{\pi^{m-1} \operatorname{vol}\left(\mathbb{S}^{m}\right)} \int_{0}^{\left(c \sqrt{\kappa_{1}}\right) / 2} \theta^{m-1} d \theta=\frac{c^{m}\left(\kappa_{1}\right)^{m / 2} \operatorname{vol}\left(\mathbb{S}^{m-1}\right)}{2 m \pi^{m-1} \operatorname{vol}\left(\mathbb{S}^{m}\right)} .
$$

Let $\kappa_{2}$ be a positive number such that $\operatorname{Ric}_{N} \geq(n-1) \kappa_{2}$. We also obtain from the Bishop inequality that

$$
v_{N}(2 c) \leq \frac{v_{\mathbb{S}^{n}}\left(2 c \sqrt{\kappa_{2}}\right)}{a_{N}\left(\kappa_{2}\right)^{n / 2}}=\frac{\operatorname{vol}\left(\mathbb{S}^{n-1}\right)}{a_{N}\left(\kappa_{2}\right)^{n / 2} \operatorname{vol}\left(\mathbb{S}^{n}\right)} \int_{0}^{2 c \sqrt{\kappa_{2}}} \sin ^{n-1} \theta d \theta<\frac{(2 c)^{n} \operatorname{vol}\left(\mathbb{S}^{n-1}\right)}{n a_{N} \operatorname{vol}\left(\mathbb{S}^{n}\right)}
$$

Recall that $\operatorname{vol}\left(\mathbb{S}^{n}\right)=2 \pi^{(n+1) / 2} / \Gamma((n+1) / 2)$. Therefore, combining the above calculations with Lemma 3.2, we complete the proof. 
Remark 3.5. In the proof of Lemma 3.4, we use only the Bishop inequality and the Bishop-Gromov volume comparison theorem. Therefore, Lemma 3.4 also holds for general mm-spaces satisfying the Bishop inequality and the Bishop-Gromov volume comparison theorem.

Proof of Proposition 1.1. Without loss of generality, it may be assumed that $n_{k} \geq$ $m_{k}$.

First, we consider the case of $\left\{\mathbb{S}^{n}\right\}_{n=1}^{\infty}$. From the assumption, we have $c^{n_{k}-m_{k}} \leq$ $c^{C_{3} k}$ for any $0<c<1$. Substituting $n:=n_{k}$ and $m:=m_{k}$, we estimate the right-hand side of the inequality of Lemma 3.4 by

$$
(1-c) \frac{n_{k} \Gamma\left(\frac{m_{k}+1}{2}\right) \Gamma\left(\frac{n_{k}}{2}\right)}{m_{k} 2^{n_{k}} \pi^{m_{k}-1} \Gamma\left(\frac{m_{k}}{2}\right) \Gamma\left(\frac{n_{k}+1}{2}\right)} \geq(1-c) 2^{-C_{1} k} \pi^{-C_{2} k+1} \frac{n_{k} \Gamma\left(\frac{n_{k}}{2}\right)}{m_{k} \Gamma\left(\frac{n_{k}+1}{2}\right)} .
$$

Therefore, if

$$
c \leq\left\{(1-c) \frac{n_{k} \Gamma\left(\frac{n_{k}}{2}\right)}{m_{k} \Gamma\left(\frac{n_{k}+1}{2}\right)}\right\}^{\frac{1}{C_{3} k}} 2^{-\frac{C_{1}}{C_{3}}} \pi^{-\frac{C_{2}}{C_{3}}+\frac{1}{C_{3} k}},
$$

then we obtain from Lemma 3.4 that $\square_{1}\left(\mathbb{S}^{n_{k}}, \mathbb{S}^{m_{k}}\right) \geq c$. Since

$$
\left\{(1-c) \frac{n_{k} \Gamma\left(\frac{n_{k}}{2}\right)}{m_{k} \Gamma\left(\frac{n_{k}+1}{2}\right)}\right\}^{\frac{1}{C_{3}}} \rightarrow 1 \text { as } k \rightarrow \infty,
$$

we have completed the proof for $\left\{\mathbb{S}^{n}\right\}_{n=1}^{\infty}$.

Next, we consider $\left\{\mathbb{C} P^{n}\right\}_{n=1}^{\infty}$. It is well-known that $\operatorname{vol}\left(\mathbb{C} P^{n}\right)=\pi^{n} / n$ ! and the sectional curvature of $\mathbb{C} P^{n}$ is bounded from below by 1 (cf. [3, Section 3.D.2, 3.H.3]). Hence, we get

$$
a_{\mathbb{C} P^{n}}=\frac{\Gamma\left(n+\frac{1}{2}\right)}{2 \sqrt{\pi} n !} .
$$

For any $0<c<1$, we have $c^{2 n_{k}-2 m_{k}} \leq c^{2 C_{3} k}$. Substituting $n:=2 n_{k}$ and $m:=$ $2 m_{k}$, we calculate the right-hand side of the inequality of Lemma 3.4 by

$$
(1-c) \frac{\Gamma\left(m_{k}+\frac{1}{2}\right)}{2 \sqrt{\pi} m_{k} 2^{2 n_{k}+1} \pi^{2 m_{k}-1} \Gamma\left(m_{k}\right)} \geq(1-c) \frac{1}{2 \sqrt{\pi} C_{2} k} \cdot 2^{-2 C_{1} k-1} \pi^{-2 C_{2} k+1} .
$$

So, if

$$
c \leq\left\{(1-c) \frac{1}{2 \sqrt{\pi} C_{2} k}\right\}^{\frac{1}{2 C_{3} k}} 2^{-\frac{C_{1}}{C_{3}}-\frac{1}{2 C_{3} k}} \pi^{-\frac{C_{2}}{C_{3}}+\frac{1}{2 C_{3} k}}
$$

then we get by using Lemma 3.4 that $\square_{1}\left(\mathbb{C} P^{n_{k}}, \mathbb{C} P^{m_{k}}\right) \geq c$. Since

$$
\left\{(1-c) \frac{1}{2 \sqrt{\pi} C_{2} k}\right\}^{\frac{1}{2 C_{3} k}} \rightarrow 1 \text { as } k \rightarrow \infty
$$

we complete the proof of the proposition.

Lemma 3.6 (J. Christensen, cf. [6, Section 3.3]). Let $X$ be a metric space and $\mu, \nu$ be uniformly distributed Borel measures on $X$. Then, there exists a positive number $c>0$ such that $\mu=c \nu$.

Proof of Proposition 1.3. We identify $\mathbb{S}^{n-1}$ with $\left\{\left(x_{1}, \cdots, x_{n}, 0\right) \in \mathbb{S}^{n} \mid\left(x_{1}, \cdots, x_{n}\right)\right.$ $\left.\in \mathbb{S}^{n-1}\right\}$. Given an arbitrary $\varepsilon>0$, since the sequence $\left\{\mathbb{S}^{n}\right\}_{n=1}^{\infty}$ is a Lévy family, we have $r_{n}:=\mu_{n}\left(\left(\mathbb{S}^{n-1}\right)_{\varepsilon}\right) \rightarrow 1$ as $n \rightarrow \infty$. Hence, there is $m \in \mathbb{N}$ such that $1-r_{n}<\varepsilon$ for any $n \geq m$. Suppose that $n \geq m$. Taking two parameters $\Phi_{1}:\left[0, r_{n}\right] \rightarrow\left(\mathbb{S}^{n-1}\right)_{\varepsilon}$ 
and $\Phi_{2}:\left(r_{n}, 1\right] \rightarrow \mathbb{S}^{n} \backslash\left(\mathbb{S}^{n-1}\right)_{\varepsilon}$, we define a Borel measurable map $\Phi:[0,1] \rightarrow \mathbb{S}^{n}$ by

$$
\Phi(t):= \begin{cases}\Phi_{1}(t), & t \in\left[0, r_{n}\right] \\ \Phi_{2}(t), & t \in\left(r_{n}, 1\right] .\end{cases}
$$

The map $\Phi$ is a parameter of $\mathbb{S}^{n}$. Let $\psi: \mathbb{S}^{n} \backslash\{(0, \cdots, 0,1),(0, \cdots, 0,-1)\} \rightarrow$ $\mathbb{S}^{n-1}$ be the projection; that is, $\psi(x)$ is the unique element of $\mathbb{S}^{n-1}$ satisfying $d_{n}(x, \psi(x))=d_{n}\left(x, \mathbb{S}^{n-1}\right)$. Put $\varphi_{1}:=\psi \circ \Phi_{1}:\left[0, r_{n}\right] \rightarrow \mathbb{S}^{n-1}$.

Claim 3.7. $\varphi_{1 *}(\mathcal{L})=r_{n} \mu_{n-1}$.

Proof. Take any Borel subset $A \subseteq \mathbb{S}^{n-1}$. For any $g \in S O(n-1)$, we have

$$
\varphi_{1 *}(\mathcal{L})(g A)=r_{n} \mu_{n}\left(\psi^{-1}(g A)\right)=r_{n} \mu_{n}\left(\psi^{-1}(A)\right)=\varphi_{1 *}(\mathcal{L})(A) .
$$

Hence, $\varphi_{1 *}(\mathcal{L})$ is an $S O(n-1)$-invariant Borel measure. From Lemma 3.6, we complete the proof of the claim.

Taking a parameter $\phi:(0,1] \rightarrow \mathbb{S}^{n-1}$ of $\mathbb{S}^{n-1}$, we define a Borel measurable map $\varphi_{2}:\left(r_{n}, 1\right] \rightarrow \mathbb{S}^{n-1}$ by $\varphi_{2}(t):=\phi\left(\left(t-r_{n}\right) /\left(1-r_{n}\right)\right)$. Then, we have $\varphi_{2 *}(\mathcal{L})=$ $\left(1-r_{n}\right) \mathcal{L}$. Therefore, defining a Borel measurable map $\varphi:[0,1] \rightarrow \mathbb{S}^{n-1}$ by

$$
\varphi(t):= \begin{cases}\varphi_{1}(t), & t \in\left[0, r_{n}\right], \\ \varphi_{2}(t), & t \in\left(r_{n}, 1\right],\end{cases}
$$

we see that the map $\varphi$ is a parameter of $\mathbb{S}^{n-1}$. Since

$$
\begin{aligned}
& \left|d_{n}(\Phi(s), \Phi(t))-d_{n-1}(\varphi(s), \varphi(t))\right| \\
= & \left|d_{n}\left(\Phi_{1}(s), \Phi_{1}(t)\right)-d_{n-1}\left(\varphi_{1}(s), \varphi_{1}(t)\right)\right| \leq 2 \varepsilon
\end{aligned}
$$

for any $s, t \in\left[0, r_{n}\right]$, we get

$$
\square_{1}\left(\mathbb{S}^{n}, \mathbb{S}^{n-1}\right) \leq \square_{1}\left(\Phi^{*} d_{n}, \varphi^{*} d_{n-1}\right) \leq \max \left\{2 \varepsilon, 1-r_{n}\right\}=2 \varepsilon .
$$

Consequently, we obtain $\square_{1}\left(\mathbb{S}^{n}, \mathbb{S}^{n-1}\right) \rightarrow 0$ as $n \rightarrow \infty$. A similar argument shows that $\square_{1}\left(\mathbb{C} P^{n}, \mathbb{C} P^{n-1}\right) \rightarrow 0$ as $n \rightarrow \infty$. This completes the proof of Proposition 1.3 .

Lemma 3.8. For any $n, m \in \mathbb{N}$, we have

$$
\square_{1}(S O(n), S O(m)) \geq c(n, m):=\min \left\{\frac{1}{2},|\operatorname{diam} S O(n)-\operatorname{diam} S O(m)|\right\} .
$$

Proof. Suppose that $n>m$ and $\unrhd_{1}(S O(n), S O(m))<c(n, m)$. There exist a compact subset $T \subseteq[0,1]$ and two parameters $\varphi_{n}:[0,1] \rightarrow S O(n), \varphi_{m}:[0,1] \rightarrow$ $S O(m)$ such that

(1) $\mathcal{L}(T)>1-c(n, m) \geq 1 / 2$,

(2) $\left.\varphi_{n}\right|_{T}: T \rightarrow S O(n),\left.\varphi_{m}\right|_{T}: T \rightarrow S O(m)$ are continuous,

(3) $\left|d_{n}\left(\varphi_{n}(s), \varphi_{n}(t)\right)-d_{m}\left(\varphi_{m}(s), \varphi_{m}(t)\right)\right|<c(n, m)$ for any $s, t \in T$.

Claim 3.9. There exist $s_{0}, t_{0} \in T$ such that $d_{n}\left(\varphi_{n}\left(s_{0}\right), \varphi_{n}\left(t_{0}\right)\right)=\operatorname{diam} S O(n)$.

Proof. Take $A_{0}, B_{0} \in S O(n)$ such that diam $S O(n)=d_{n}\left(A_{0}, B_{0}\right)$ and define a map $\psi: S O(n) \rightarrow S O(n)$ by $\psi(A):=A A_{0}^{-1} B$. Then, $\psi_{*}\left(\mu_{n}\right)=\mu_{n}$ and $d_{n}(A, \psi(A))=$ 
$\operatorname{diam} S O(n)$ for any $A \in S O(n)$. Suppose that $d_{n}\left(\varphi_{n}(s), \varphi_{n}(t)\right)<\operatorname{diam} S O(n)$ for any $s, t \in T$. Then, we get $\psi\left(\varphi_{n}(T)\right) \cap \varphi_{n}(T)=\emptyset$, which leads to

$$
\begin{aligned}
\mu_{n}\left(\psi\left(\varphi_{n}(T)\right) \cap \varphi_{n}(T)\right) & =\mu_{n}\left(\psi\left(\varphi_{n}(T)\right)\right)+\mu_{n}\left(\varphi_{n}(T)\right) \\
& =\mu_{n}\left(\psi^{-1}\left(\psi\left(\varphi_{n}(T)\right)\right)\right)+\mu_{n}\left(\varphi_{n}(T)\right) \\
& \geq 2 \mu_{n}\left(\varphi_{n}(T)\right)>1 .
\end{aligned}
$$

This is a contradiction, and thus we complete the proof of the claim.

By Claim 3.9, we obtain

$\operatorname{diam} S O(n)-\operatorname{diam} S O(m) \leq\left|d_{n}\left(\varphi_{n}\left(s_{0}\right), \varphi_{n}\left(t_{0}\right)\right)-d_{m}\left(\varphi_{m}\left(s_{0}\right), \varphi_{m}\left(t_{0}\right)\right)\right|<c(n, m)$, which is a contradiction. This completes the proof of Lemma 3.8.

Proof of Proposition 1.2. An easy calculation shows that $2 \sqrt{n-1} \leq \operatorname{diam} S O(n) \leq$ $2 \sqrt{n}$. Therefore, supposing $n_{k} \geq m_{k}$, we have

$$
\begin{aligned}
\operatorname{diam} S O\left(n_{k}\right)-\operatorname{diam} S O\left(m_{k}\right) & \geq 2 \sqrt{n_{k}-1}-2 \sqrt{m_{k}} \\
& =2 \frac{n_{k}-m_{k}-1}{\sqrt{n_{k}-1}+\sqrt{m_{k}}} \geq 2 \frac{C_{3}-1 / \sqrt{k}}{\sqrt{C_{1}-1 / k}+\sqrt{C_{2}}} .
\end{aligned}
$$

Thus, applying Lemma 3.8 , we complete the proof.

\section{ACKNOWLEDGEMENTS}

The author would like to thank Professor Takashi Shioya and Mr. Masayoshi Watanabe for valuable discussions and many fruitful suggestions. He also thanks Professor Hajime Urakawa for his encouragement.

\section{REFERENCES}

1. T. H. Colding, Large manifolds with positive Ricci curvature, Invent. Math. 124, no. 1-3, pp. 193-214, 1996. MR1369415 (96k:53068)

2. K. Funano, A note for Gromov's distance functions on the space of $\mathrm{mm}$-spaces, available online at http://front.math.ucdavis.edu/0706.2647

3. S. Gallot, D. Hulin, J. Lafontaine, Riemannian geometry, Third edition, Springer-Verlag, Berlin, 2004. MR2088027 (2005e:53001)

4. M. Gromov, Metric structures for Riemannian and non-Riemannian spaces. Based on the 1981 French original, with appendices by M. Katz, P. Pansu and S. Semmes. Translated from the French by Sean Michael Bates. Progress in Mathematics, 152. Birkhäuser Boston, Inc., Boston, MA, 1999. MR1699320(2000d:53065)

5. M. Gromov, V. D. Milman, A topological application of the isoperimetric inequality, Amer. J. Math. 105, no. 4, pp. 843-854, 1983. MR708367 (84k:28012)

6. P. Mattila, Geometry of sets and measures in Euclidean spaces. Fractals and rectifiability, Cambridge Studies in Advanced Mathematics, 44. Cambridge University Press, Cambridge, 1995. MR1333890(96h:28006)

Mathematical Institute, Tohoku University, Sendai 980-8578, Japan

E-mail address: sa4m23@math.tohoku.ac.jp 